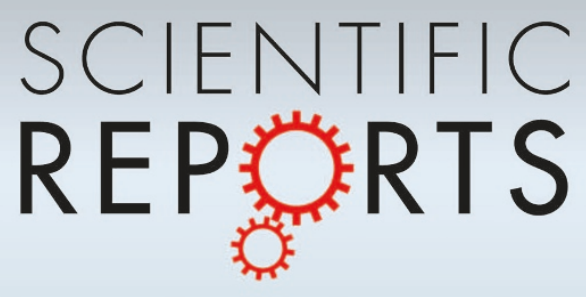

OPEN

CONFERENCE PROCEEDINGS

Symposium N + O

E-MRS 2012

Spring Meeting

..............

SUBJECT AREAS:

OPTICAL MATERIALS AND

STRUCTURES

CONDENSED-MATTER PHYSICS

NANOPARTICLES

OPTICAL MATERIALS

Received

3 September 2012

Accepted

19 October 2012

Published

3 December 2012

Correspondence and requests for materials should be addressed to A.A. (alu@mail.utexas. edu)

\section{Layered plasmonic cloaks to tailor the optical scattering at the nanoscale}

\author{
F. Monticone, C. Argyropoulos \& A. Alù
}

Department of Electrical and Computer Engineering, The University of Texas at Austin, Austin, TX 78712, USA.

We discuss the rich scattering features offered by thin and thick plasmonic layers covering dielectric nanoparticles and their potential optical applications. The frequency position of scattering dips and peaks may be controlled to a large degree using plasmonic layers, which may dramatically vary the total scattering signature in the frequency range of interest. We show that complex and exotic spectra may be obtained using a single composite nanosphere, including dipole-dipole Fano resonances and electromagnetic induced transparency effects. The described phenomena are observable at any angle of observation, in the total scattering cross section and for realistic plasmonic materials, since they are based on purely dipolar fields. Enhanced field amplitudes are associated with these anomalous scattering features, which may be used to efficiently boost weak optical nonlinear effects. Exciting applications of these nanostructures are envisioned, such as efficient and tunable sensors, all-optical switches and memories, optical tagging and biomolecular imaging.

ecently, large interest has been dedicated to the study of plasmonic materials at terahertz, infrared (IR) and optical frequencies ${ }^{1}$. At the interface with air or with other dielectrics, these materials support localized resonances associated with surface plasmons, of great interest for imaging and energy harvesting applications $^{2}$. Specific arrangements of plasmonic and layered nanoparticles may support resonances with sharp spectral signatures and large near-field intensities, associated with Fano resonances ${ }^{3-7}$ and electromagnetic induced transparency (EIT) effects ${ }^{8-10}$. In general, these extreme resonant phenomena are generated by the strong interaction and coupling between bright and dark resonant modes supported by a complex subwavelength plasmonic system. It has been suggested that these exotic scattering responses may have a plethora of practical applications, spanning from 'slow light' devices to novel sensing systems.

In a different context, plasmonic coatings have been used in a nonresonant way to suppress the electric dipole scattering from small dielectric or conducting objects, leading to the development of 'plasmonic cloaking' devices $^{11-13}$. This concept has been recently experimentally verified at microwave frequencies using plasmonic metamaterials $s^{14,15}$ and at $\operatorname{IR}^{16}$ frequencies.

It is interesting that plasmonic features can effectively lead to dual scattering phenomena, from very large and enhanced scattering signatures ${ }^{17-19}$ to very low and suppressed response. Hence, exploiting the natural dispersion of plasmonic materials we may be able to design a plasmonic nanoparticle configuration supporting low-scattering cloaked states followed in close frequency range by highly resonant scattering peaks. In this work we explore whether we may combine and control these effects with plasmonic covers, in order to tailor to a large degree the complex scattering response of small nanoparticles, spanning Fano, EIT-like, super-resonant and cloaking features in a single nanoparticle.

We limit our analysis to subwavelength spherical nanoparticles, for which the electric dipole response dominates the overall scattering. Resonant dipolar scattering is generally characterized by a peak with relatively broad linewidth. In conventional plasmonic Fano resonances ${ }^{3-6}$, this resonance is typically interpreted as the 'bright' bonding mode that may be coupled to a narrowband higher-order 'dark' antibonding scattering mode, typically an electric quadrupole or higher-order multipole, to generate the typically antisymmetric Fano signature. As we have briefly discussed in Ref. 20, however, we may alternatively exploit the 'dark' state associated with the plasmonic cloaking scheme ${ }^{11}$ to achieve similar sharp scattering responses. This novel mechanism for Fano signatures has a different physical nature compared to the conventional antibonding resonant modes, since it exhibits lower levels of stored electromagnetic energy and a response that is associated with only dipolar scattered fields, and is therefore directly reflected into the total scattering signature of the object.

In the following, we explore and expand on the concept of Fano-like resonances in realistic composite plasmonic nanoparticles, based only on the coupling and interaction of dipolar 'bright' and 'dark' scattering states. These dipole-dipole Fano-like resonances have been recently introduced for concentric ${ }^{21,22}$, single coated ${ }^{20}$ 
and multi-layered ${ }^{23}$ geometries. It has been shown that they can support strongly confined fields at the nanoparticle's core, which is an ideal condition to boost third-order optical nonlinearities to realize efficient all-optical nanoswitches ${ }^{20,24}$. In the following, single- and double-layered nanospheres are theoretically investigated and explored. It is shown that they provide unprecedented flexibility in the design and manipulation of the total scattering response as a function of the thickness and number of plasmonic layers. We further demonstrate that, even with purely symmetric and isotropic nanoparticles, it is possible to tailor to a large extent the position of zeros and poles of their scattering response and interesting degenerate states may be induced, such as ultrasharp Fano resonances and EIT scattering signature, without requiring complex asymmetric plasmonic configurations. Some of the concepts presented in this report have been briefly discussed in Refs. 20-23 for different purposes and applied to specific configurations. In Ref. 20 we exploited a simple degenerate state supported by a core-shell particle to realize all-optical nanoswitches, whereas in Ref. 23 we analyzed the case of a four-layer particle supporting a comb-like spectrum with super-scattering features, which is well suited for optical tagging applications. Differently from all these previous works, here we discuss and investigate more generally the potential of plasmonic cloaks to tailor the scattering response and obtain exotic and useful scattering features.
We also explore their evolution when design parameters and losses are considered. These results may have exciting implications for the design of advanced photonic and sensing nanodevices.

\section{Results}

Single layer plasmonic composite nanoparticle. In order to understand the possibilities offered by plasmonic cloaks in terms of scattering manipulation, we start by considering the basic geometry shown in Fig 1(a). It consists of a simple spherical nanoparticle composed of a dielectric core (yellow region) with relative permittivity $\varepsilon=2$ and radius $a$ coated by a plasmonic shell (gray region) of radius $a_{c}$. In order to gain physical insights into the dependence of the scattering properties on the design parameters of this nanoparticle, we start by analyzing the ideal lossless scenario in which the plasmonic shell is assumed lossless, following a Drude permittivity dispersion with parameters $\varepsilon_{c}=\varepsilon_{\infty}-f_{p}^{2} /[f(f+i \gamma)]$, $f_{p}=2175 \mathrm{THz}, \gamma=0 \mathrm{THz}, \varepsilon_{\infty}=5$, fitted to the experimental data for the real part of silver permittivity ${ }^{25}$, under an $e^{-i \omega t}$ time convention. Although the lossless case will be useful to gain insights into the proposed phenomena, their realistic implementation require addressing the issue of losses, which is usually critical in plasmonic effects. To assess this important aspect, we also consider in the following realistic silver absorption in several cases, corresponding
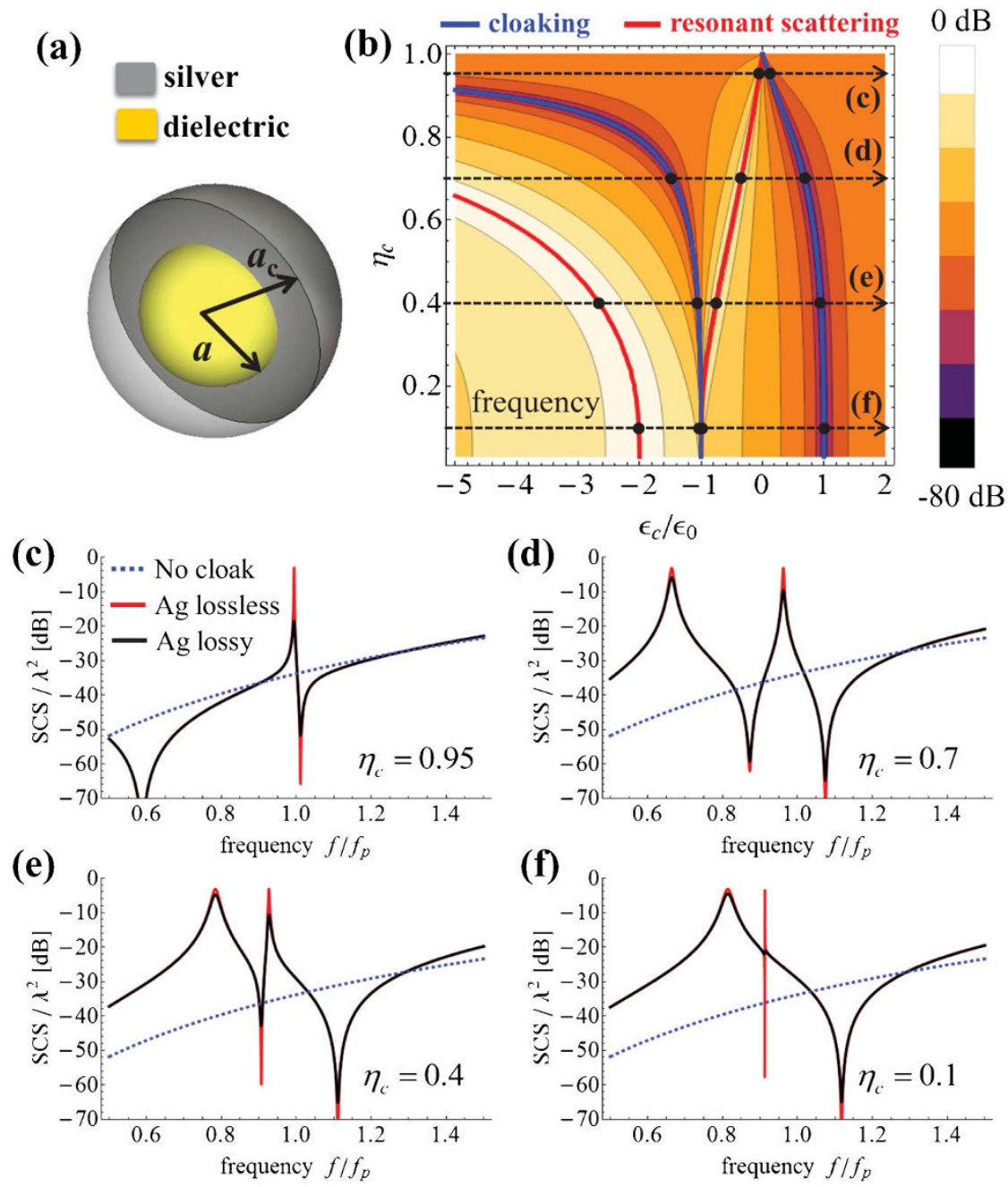

Figure 1 SCS evolution of a core-shell plasmonic nanoparticle with different aspect ratios. (a) Geometry of a core-shell plasmonic nanosphere. The dielectric core has relative permittivity $\varepsilon=2$ and the outer radius is fixed to $a_{c}=30 \mathrm{~nm}$ in all the considered examples. (b) Contour plot of the normalized SCS as a function of the relative permittivity of the plasmonic coating $\varepsilon_{c} / \varepsilon_{0}$ and the aspect ratio $\eta_{c}=a / a_{c}$. Cloaking (blue lines) and resonant scattering (red lines) quasi-static conditions are also shown. (c-f) Normalized SCS versus normalized frequency are shown for lossless (red solid line) and lossy (black solid line) plasmonic covers for different aspect ratios: (c) $\eta_{c}=0.95$, (d) $\eta_{c}=0.7$, (e) $\eta_{c}=0.4$ and (f) $\eta_{c}=0.1$. 
to a collision frequency $\gamma=4.35 \mathrm{THz}^{25}$. This will allow assessing the sensitivity of the proposed concepts to material losses.

It is convenient to analyze the scattering response of this spherical core-shell geometry by applying the conventional Mie theory ${ }^{17}$. As usual, the fields become a discrete sum of orthogonal transverse magnetic (TM) and transverse electric (TE) spherical harmonics. The $\mathrm{n}$-th order TM scattering coefficient $c_{n}^{T M}$ is given as

$$
c_{n}^{T M}=-\frac{U_{n}^{T M}}{U_{n}^{T M}+i V_{n}^{T M}},
$$

where the complex valued quantities $U_{n}^{T M}, V_{n}^{T M}$ can be calculated by solving the appropriate determinants ${ }^{11,18}$ and take real values in the lossless case. By duality, one can straightforwardly compute the TE complex scattering coefficients $c_{n}^{T E}$. The total SCS is computed as the sum of TM and TE scattering coefficients and it is equal to

$$
S C S=\frac{\lambda_{0}^{2}}{2 \pi} \sum_{n=1}^{\infty}(2 n+1)\left(\left|c_{n}^{T M}\right|^{2}+\left|c_{n}^{T E}\right|^{2}\right),
$$

where $\lambda_{0}$ is the free-space wavelength. We assume that the composite nanoparticle is smaller than the radiation wavelength and has no inherent magnetic effects, implying that the TM spherical harmonic with $n=1$ dominates Eq. (2), corresponding to electric dipole radiation. It is usually assumed that, under the condition that only one scattering order dominates the total SCS, the scattering spectra may not be particularly exotic. However, one may notice that when $U_{1}^{T M}$ is set to zero, Eq. (1) vanishes and the total SCS may become very small, consistent with the cloaking condition described in Ref. 11. Conversely, when $V_{1}^{T M}=0, c_{n}^{T M}=-1$ and maximum scattering is obtain$\mathrm{ed}^{18}$. If we are able to tailor the position of the zeros of these two parameters in the desired frequency range, we may be able to realize complex scattering features from such simple symmetric nanoparticle, even in the quasi-static limit.

As noticed in Ref. 20, in the long-wavelength regime the quasistatic condition to achieve cloaking $\left(U_{1}^{T M}=0\right)$ depends only on the filling ratio $\eta_{c}=a / a_{c}$ of the nanoparticle and its material properties:

$$
\eta_{c}^{3}\left(\varepsilon-\varepsilon_{c}\right)\left(2 \varepsilon_{c}+1\right)+\left(\varepsilon+2 \varepsilon_{c}\right)\left(\varepsilon_{c}-1\right)=0,
$$

Similarly, resonant scattering $\left(V_{1}^{T M}=0\right)$ is obtained when the filling ratio satisfies ${ }^{20}$

$$
2 \eta_{c}^{3}\left(\varepsilon-\varepsilon_{c}\right)\left(\varepsilon_{c}-1\right)+\left(\varepsilon+2 \varepsilon_{c}\right)\left(\varepsilon_{c}+2\right)=0
$$

We show in Fig. 1(b) the contour plot of the total SCS of the proposed nanoparticle with $\varepsilon=2$ and fixed outer radius $a_{c}=30 \mathrm{~nm}$ as a function of the aspect ratio $\eta_{c}$ and of the relative permittivity $\varepsilon_{c}$ of the plasmonic coating (brighter colors correspond to larger SCS). In this panel, we consider the lossless scenario only. The dispersive permittivity $\varepsilon_{c}$ increases monotonically with frequency, as dictated by Kramers-Kronig relations and indicated by the horizontal dashed arrows in Fig. 1(b). The dispersion of the solutions of Eqs. (3)-(4) is also plotted in Fig. 1(b), as the solid blue (cloaking) and red (resonant scattering) lines, respectively.

In Figs. 1(c)-(f), we present the evolution of the normalized total SCS spectra (solid red lines) for different aspect ratios, as indicated by the different horizontal dashed lines in Fig. 1(b). The total SCS of a bare dielectric object (dashed blue line) with same outer radius as the considered nanoparticle is also plotted in Figs. 1(c)-(f) for comparison. It is interesting to see how the intersections of the dashed lines in Fig. 1(b) with the blue and red dispersion curves (zeros of $U_{1}^{T M}$ and $V_{1}^{T M}$, respectively) are directly reflected in dips and peaks in the total SCS. When the location of these zeros gets closer in frequency, anomalous scattering features are observed. Figure 1(c) shows the scattering response of a nanoparticle with large aspect ratio $\left(\eta_{c}=0.95\right)$, which corresponds to a thin plasmonic shell. In this case, a rather broadband low scattering (cloaked) region is observed at low frequencies, similar to the conventional plasmonic cloaking scheme $^{11}$. At higher frequencies, a second cloaking condition lies in close proximity to a resonant state [see Fig. 1(b)], producing a very peculiar asymmetric response. This is a first example of degenerate state between cloaking and resonant scattering, which resembles the shape of a Fano resonance. We stress that this is rather different from conventional Fano resonances, in which different orthogonal scattering orders interfere within the angular spectrum. Here, the same scattering order (electric dipolar) is responsible for this anomalous scattering response. Figure 1(d) shows the SCS spectrum for a plasmonic nanoparticle with aspect ratio $\eta_{c}=0.7$. In this case, two distinct frequency regions of suppressed scattering are obtained and the nanoparticle can be effectively transparent in two different frequency bands ${ }^{12}$. However, a high resonant scattering peak always exists between the two scattering dips. This is a general constraint when considering causality and passivity limitations of plasmonic materials, which prevents the possibility of realizing a passive broadband cloaking device based on merging two cloaked states along the frequency axis ${ }^{12,23}$. Always a scattering peak is expected to arise between the two dips, consistent with Foster's reactance rule for the response of passive circuit networks ${ }^{26,27}$.

For lower aspect ratios the scattering signature of the proposed plasmonic nanoparticle becomes even more intriguing and complex. The SCS for $\eta_{c}=0.4$ is shown in Fig. 1(e) and again a relative broad cloaking frequency region is observed, now in the higher frequency range. More interestingly, an additional asymmetric scattering resonance is obtained at lower frequencies, due to the interference between the closely spaced cloaking dip and scattering peak. As in panel (c), the merging of the cloaking and resonant states gives rise to a dipole-dipole Fano-like resonance ${ }^{20-23}$, which can have extremely narrow linewidth for even lower aspect ratios, as observed in Fig. 1(f) for $\eta_{c}=0.1$. The linewidth is ultimately limited by losses in the materials and quantum effects that bound how small we can make the inner core without affecting the resonant state. To quantitatively assess the sensitivity to realistic material absorption, we also show the SCS of the composite particle when the real losses are considered [black curves in panels (c)-(f)]. It is evident that absorption affects the scattering signature, by reducing the scattering excursion, but the general behavior and the most interesting features are maintained. It is interesting that the asymmetric resonances can retain a very sharp linewidth, except when the cloaking and resonance states are too close in frequency, as in panel (f). This remarkable robustness to losses is due to the purely dipolar nature of the proposed effect.

It is important to stress that, different from conventional plasmonic Fano-resonances ${ }^{3}$ based on the interaction of 'bright' dipolar with 'dark' higher-order modes, this dipole-dipole Fano-like response can be observed for any angle of observation and it is detectable in the total SCS signature, due to the isotropy of the plasmonic nanoparticle and the dipolar nature of both bonding and antibonding states involved.

To further study these interesting phenomena, we explicitly calculate the field distributions for $\eta_{c}=1 / 3$ at specific frequencies of interest for the lossless geometry. The normalized SCS versus wavelength is plotted in Fig. 2(b) in the lossless case. The points I and IV in Fig. 2(b) are the usual, moderately broad cloaking and resonant scattering states of the plasmonic nanoparticle, respectively. Instead, the points II and III in Fig. 2(b) correspond to an extremely narrowband asymmetric resonance with an almost $50 \mathrm{~dB}$ abrupt change between cloaking and high scattering states. As explained in the previous paragraphs, the asymmetric Fano-like scattering response is a signature of the degenerate state between these two distinct dipolar phenomena.

The corresponding electric field distributions on the $\mathrm{E}$ plane are plotted in Figs. 2(c) (snapshot in time of the $\mathrm{E}_{\mathrm{x}}$ component, parallel to the incident electric field) and (d) (magnitude of the total electric field) for all these frequency points. In addition, the arrows in panel (d) represent the power flow (time-average Poynting vector) 
(a)

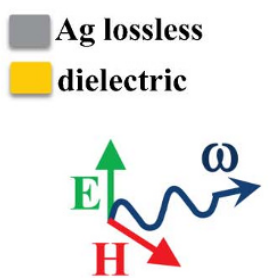

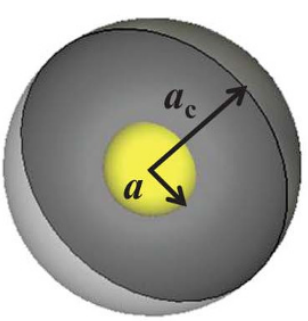

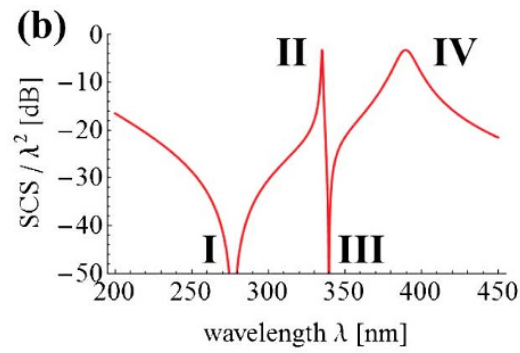

(c)
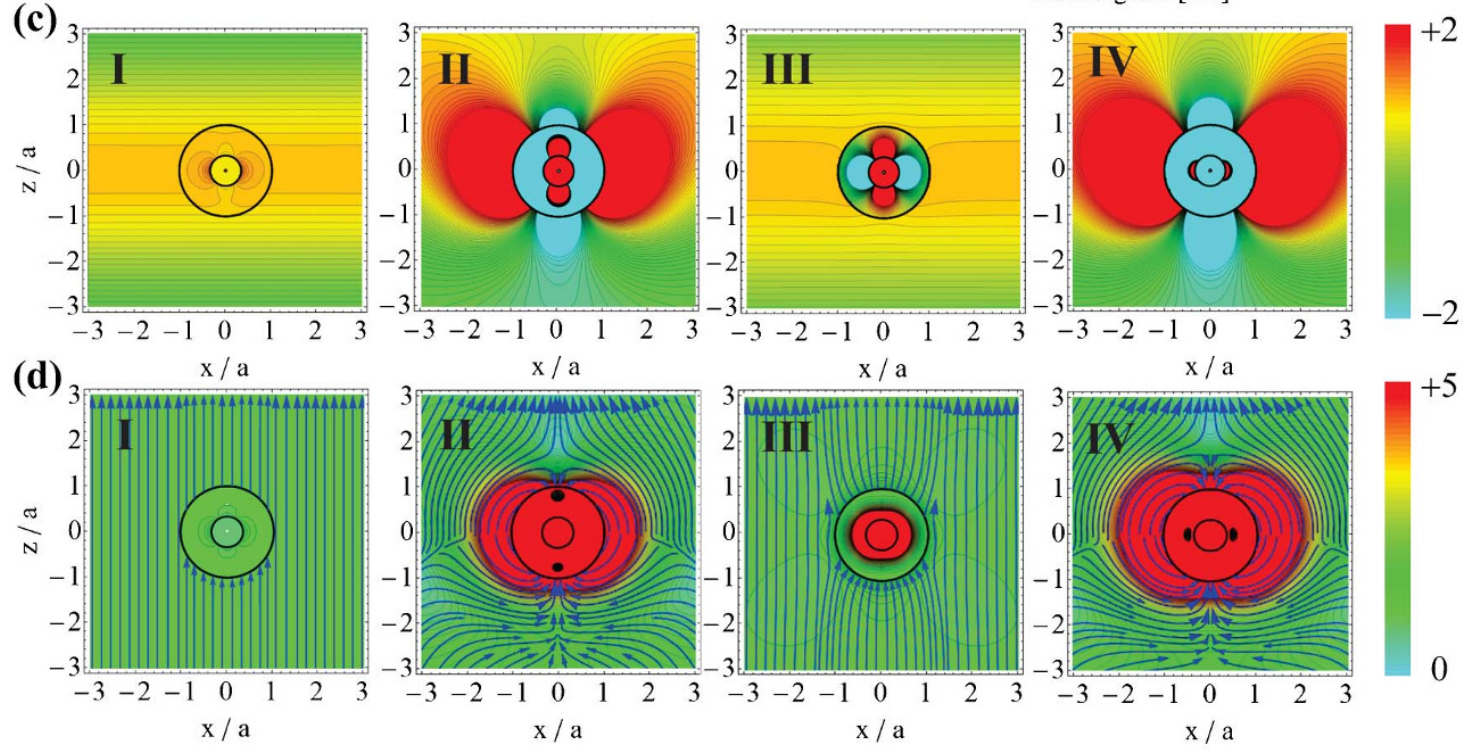

Figure $2 \mid$ Field plots of a core-shell plasmonic nanoparticle. (a) Geometry of the plasmonic nanosphere, with outer radius $a_{c}=30 \mathrm{~nm}$, aspect ratio $\eta_{c}=1 / 3$ and $\varepsilon=2$. b) Normalized SCS versus wavelength in the lossless case. (c) Corresponding electric field plots for the real part of the $\mathrm{E}_{\mathrm{x}}$ component (snapshot in time) and (d) the amplitude of the total electric field, in the E plane at the broad cloaking dip (I), the narrow Fano-like resonant peak (II), the narrow Fano-like cloaking dip (III) and the broad resonant peak (IV). Blue arrows in (d) represent the corresponding power flow (time-average Poynting vector) distributions. The impinging plane wave travels from bottom to top with electric field linearly polarized along x. Amplitude levels in all panels are normalized to the impinging wave.

distribution around the nanoparticle. The overall field distributions at points II and IV show a clearly dipolar resonant response. The corresponding power flow around the particle is very complex, since the SCS is much larger than the physical cross section of the particle and typical vortices and saddle points are observed in the vicinity of the particle ${ }^{28,29}$. The fields in the core are very much enhanced at these two wavelengths, as expected for a resonant state. At the other two wavelengths of interest, points I and III, a dual scattering response is observed: very low scattering, i.e., cloaking, is achieved right around the nanoparticle and the impinging plane wave fields are effectively restored, as if the particle was transparent. By comparing the two cloaked cases, however, a crucial difference may be observed: at the broad cloaking frequency (point I), the field amplitude is also very low inside the core, comparable to the impinging levels; conversely the fields are strongly enhanced and confined at the particle's core at the Fano dip (point III). This is due to the close interaction of the cloaked and resonant states in this latter case, which produces the exotic situation in which the fields are almost unperturbed outside the cloak, but strongly enhanced inside it. This phenomenon can lead to efficient sensors and enhanced nonlinear effects ${ }^{20}$. It is remarkable that in a small frequency window (points II and III), the field distribution outside the nanoparticle can be dramatically altered from invisible to a bright scattering state, while the interior fields are drastically enhanced. These interesting properties, combined with enhanced third-order nonlinear effects, may potentially lead to highly efficient all-optical nanoswitches and memories ${ }^{20}$.

Double-layer plasmonic composite nanoparticle. After having established that a simple plasmonic layer can produce exciting scattering features, we show in this section that even more complicated scattering signatures may be designed and tailored using multilayered cloaks for a single, isotropic subwavelength nanoparticle. As we show in the following, multiple Fano-like resonances and other complex scattering features may be produced using two different plasmonic coating layers, further enriching the nanoparticle's scattering spectrum. The geometry of this configuration is shown in Fig. 3(a): the dielectric core has a relative permittivity $\varepsilon_{1}=2$ and the outer radius of the composite nanoparticle is fixed at $a_{c}=50 \mathrm{~nm}$. The dispersion of the first plasmonic layer follows the same Drude permittivity, and we consider both the lossless $(\gamma=0 \mathrm{THz})$ and lossy scenarios $(\gamma=4.35 \mathrm{THz})$. The second plasmonic layer has a relative permittivity $\varepsilon_{3}$ with similar Drude dispersion, but a plasma frequency decreased by $\Delta f_{p}=325 \mathrm{THz}$. This may be achieved, for instance, by modifying the doping level of some plasmonic semiconductors, similar to the case of four-layer plasmonic nanoparticles based on aluminum-doped zinc oxide (AZO) semiconductors ${ }^{30,31}$ proposed in Ref. 23.

In this case, the quasi-static condition for resonant scattering $\left(V_{1}^{T M}=0\right)$ may be calculated using Mie theory:

$$
\begin{aligned}
& 2 \eta_{2}^{3}\left(\varepsilon_{1}-\varepsilon_{2}\right)\left[\left(\varepsilon_{2}-\varepsilon_{3}\right)\left(\varepsilon_{3}+2\right)+\eta_{1}^{3}\left(2 \varepsilon_{2}+\varepsilon_{3}\right)\left(\varepsilon_{3}-1\right)\right]+ \\
& \eta_{1}^{3}\left(\varepsilon_{1}+2 \varepsilon_{2}\right)\left[2 \eta_{1}^{3}\left(\varepsilon_{2}-\varepsilon_{3}\right)\left(\varepsilon_{3}-1\right)+\left(\varepsilon_{2}+2 \varepsilon_{3}\right)\left(\varepsilon_{3}+2\right)\right]=0,
\end{aligned}
$$

where $\eta_{1}=\left(a+t_{\text {in }}\right) /\left(a+t_{\text {in }}+t_{\text {out }}\right)$ is the aspect ratio of the inner plasmonic layer, with radius $a+t_{i n}$, over the outer plasmonic layer, with radius $a+t_{\text {in }}+t_{\text {out }}=a_{c}$. Furthermore, $\eta_{2}=a /\left(a+t_{\text {in }}+t_{\text {out }}\right)$ is the aspect ratio of the dielectric core with radius $a$ over the outer 
(a)

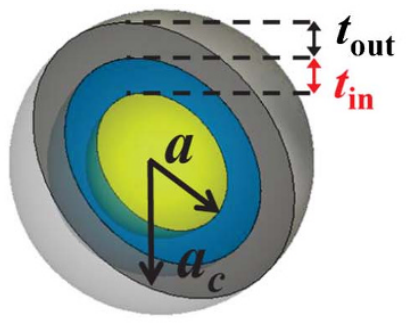

(c)

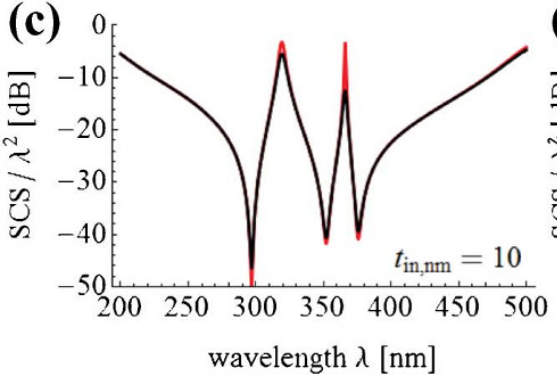

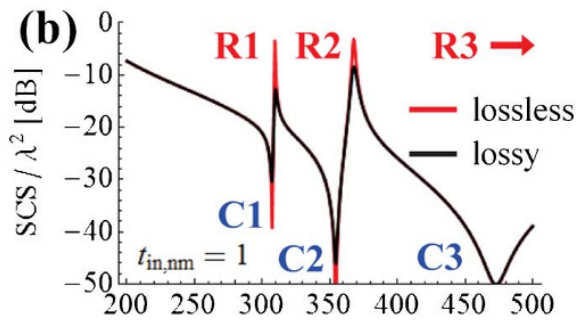

(d)

wavelength $\lambda[\mathrm{nm}]$

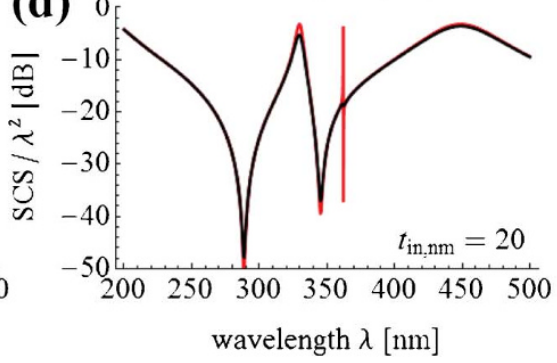

Figure $3 \mid$ SCS of a double-layer plasmonic nanoparticle with different inner layer thicknesses. (a) Geometry of the double-layered plasmonic nanosphere. The dielectric core has relative permittivity $\varepsilon_{1}=2$ and the outer radius is $a_{c}=50 \mathrm{~nm}$. The outer layer thickness is fixed to $t_{\text {out }}=5 \mathrm{~nm}$. (b), (c) and (d) show the normalized SCS for lossless (red solid line) and lossy (black solid line) plasmonic covers for three different inner layer thicknesses: $t_{i n}=1 \mathrm{~nm}, t_{i n}=10 \mathrm{~nm}$ and $t_{i n}=20 \mathrm{~nm}$, respectively.

plasmonic layer. The parameters $t_{\text {in }}$ and $t_{\text {out }}$ are the thicknesses of the inner and outer plasmonic shells, respectively. In a similar fashion, the cloaking condition $\left(U_{1}^{T M}=0\right)$ can be evaluated in the long-wavelength limit as

$$
\begin{aligned}
& \eta_{2}^{3}\left(\varepsilon_{1}-\varepsilon_{2}\right)\left[2\left(\varepsilon_{2}-\varepsilon_{3}\right)\left(\varepsilon_{3}-1\right)+\eta_{1}^{3}\left(2 \varepsilon_{2}+\varepsilon_{3}\right)\left(2 \varepsilon_{3}+1\right)\right]+ \\
& \eta_{1}^{3}\left(\varepsilon_{1}+2 \varepsilon_{2}\right)\left[\eta_{1}^{3}\left(\varepsilon_{2}-\varepsilon_{3}\right)\left(2 \varepsilon_{3}+1\right)+\left(\varepsilon_{2}+2 \varepsilon_{3}\right)\left(\varepsilon_{3}-1\right)\right]=0 .
\end{aligned}
$$

As in the previous section, these formulas can accurately describe the cloaking and resonant scattering behavior of the double-layer particle in the quasi-static limit of interest here.

In Fig. 3, we show the scattering response of this double-layer structure varying the thickness of the inner layer. While this thickness is modified, the outer radius of the nanoparticle is kept constant, i.e., we shrink the dielectric core, for fair comparison. The outer layer thickness in this study is fixed at $t_{\text {out }}=5 \mathrm{~nm}$. In this case, three distinct scattering peaks and dips may be induced in the scattering signature, visible in Figs. 3(b), (c) and (d), where the normalized SCS is plotted versus impinging wavelength for three different inner layer thicknesses: $t_{i n}=1 \mathrm{~nm}, t_{i n}=10 \mathrm{~nm}$ and $t_{i n}=20 \mathrm{~nm}$, respectively. Figure 3(b) clearly demonstrates two consecutive narrowband Fano-like resonances indicated by the labels C1, R1 and C2, R2, respectively. Each Fano-like feature is a degenerate state between neighboring cloaking and resonant scattering, generalizing the results of the previous section. We notice in the following panels that, as the inner layer thickness is increased, the scattering spectrum evolves to an interesting "inverse EIT" signature around $\lambda=360 \mathrm{~nm}$ (Panel (c) in Fig. 3), due to the strong interaction and coupling between the narrow linewidths of the two distinct Fano-like resonances. This can be considered a triple degenerate state between a resonant mode and two cloaking states. These effects are mostly preserved after adding realistic level of losses in the plasmonic layers [black curves in Fig. 3]. Finally, in Fig. 3(d), we can see how the inverse EIT profile collapses to an ultrasharp Fano resonance, as the thickness of the inner layer is further increased. However, in this case absorption strongly affects this ultranarrow resonant feature. It is exciting to observe the flexibility with which we can engineer the scattering spectrum of a simple symmetric composite nanoparticle by simply moving the zeros of $U_{1}^{T M}$ and $V_{1}^{T M}$ by varying the geometry according to Eqs. (5)-(6).
So far, we have varied only the inner layer thickness. Analogously, the thickness of the outer layer may also be used as a tool to manipulate the scattering response, as we consider in Fig. 4. The figure is analogous to Fig. 3, but in this case the inner layer thickness is fixed to $t_{i n}=5 \mathrm{~nm}$ and the scattering response is analyzed as the thickness of the outer layer is increased. As seen in panels (b), (c) and (d) of Fig. 4, also in this case the two Fano-like resonances are modified in a peculiar way as the geometry of the nanoparticle is changed. In particular, it is interesting that in Fig. 4(c) the two Fano resonances "unfold" and the scattering signature assumes a symmetric EIT-like shape around $350 \mathrm{~nm}$, unaffected by the realistic plasmonic losses, with interesting potential for multiband sensing.

It is clear from these results that dual-layer cloaks offer exciting possibilities to manipulate the scattering spectrum of small nanoparticles, even if limited to just the dominant, purely dipolar scattering order. By varying the position of the zeros of $U_{1}^{T M}$ and $V_{1}^{T M}$, we are able to position cloaked and resonant states across the scattering signature spectrum and tailor the overall response of the nanoparticle to a large degree. This is in some sense similar to the way filter responses are designed in circuit theory by manipulating the position of zeros and poles of their transfer function ${ }^{26,32}$. These results are summarized in Fig. 5, which shows the frequency variation of the different cloaking and resonant scattering conditions as a function of the inner and outer layer thicknesses. Comparing panel (a) (inner layer variation) and (b) (outer layer variation) of Fig. 5, it is interesting to note that the Fano-like resonance composed by the points $\mathrm{C} 2$ and $\mathrm{R} 2$ is more sensitive to the change of the outer layer thickness than to the inner layer one. Conversely, the Fano-like resonance arising at points $\mathrm{C} 1$ and $\mathrm{R} 1$ is affected by the thickness of the inner layer. The branches C3 and R3 are influenced by a change of both inner and outer layer thicknesses, consistent with the results in Figs. 3 and 4 . Therefore, each cloaking-resonance pair is sensitive in a different way to the geometrical parameters of the double-layer nanoparticle and this can be used to engineer the spectrum in a variety of ways. Figure 5 also provides an insight on how the Fano-like response is physically established: it is evident that the cloaking-resonance pair C1-R1 is due to the interaction of two surface plasmons on the two sides of the inner plasmonic layer, whereas the pair C2-R2 is determined by the plasmons on the outer plasmonic layers. We have verified by inspecting the field distributions at the two wavelengths 
(a)
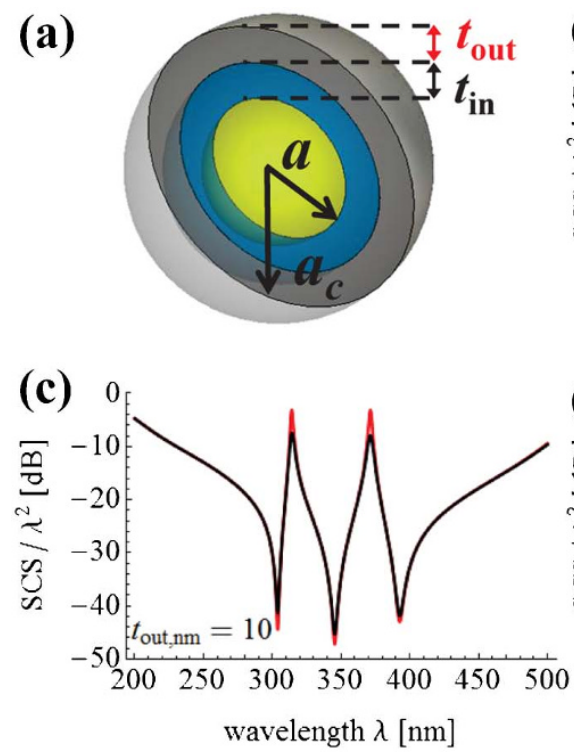
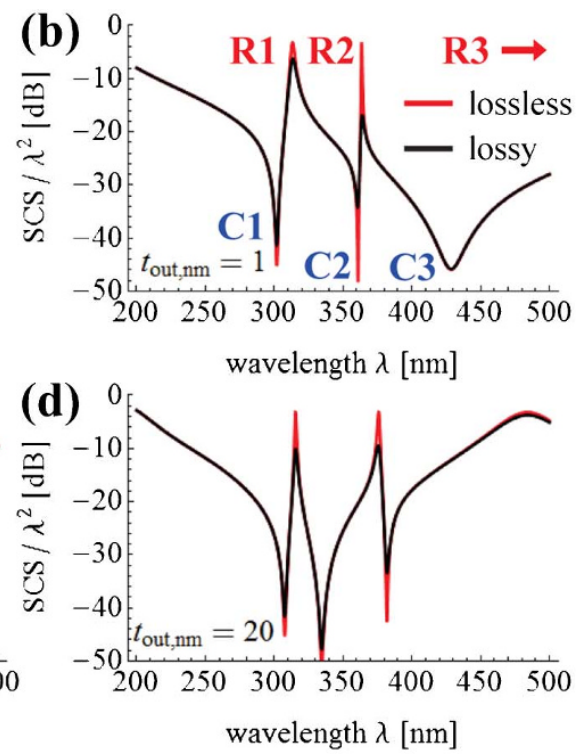

Figure $4 \mid$ SCS of a double-layer plasmonic nanoparticle with different outer layer thicknesses. (a) Geometry of the double-layered plasmonic nanosphere, similar to Fig. 3. Here the inner layer thickness is fixed to $t_{i n}=5 \mathrm{~nm}$. (b), (c) and (d) show the normalized SCS for lossless (red solid line) and lossy (black solid line) plasmonic covers for three different outer layer thicknesses: $t_{\text {out }}=1 \mathrm{~nm}, t_{\text {out }}=10 \mathrm{~nm}$ and $t_{\text {out }}=20 \mathrm{~nm}$, respectively.

that this is indeed the case (not reported here for brevity). Finally, the C3-R3 pair, sensitive to both inner and outer layer thicknesses, is based on less confined plasmon modes, which is reflected in less sharp spectral features. It is evident that the addition of a second plasmonic layer provides more degrees of freedom to the design and tailoring of the scattering response. As demonstrated here, we may achieve significantly complex scattering signatures, such as multiple Fano-like resonances which can also evolve in EIT-like responses.

\section{Discussion}

In this Report, we have demonstrated an unprecedented capability to manipulate and tailor the scattering response of a composite nanosphere with subwavelength size by using multilayered plasmonic covers. We have first investigated the simple core-shell geometry and we have shown interesting degenerate states between cloaking and resonant scattering, which give rise to ultrasharp dipole-dipole Fano resonances in the scattering spectrum. The addition of a further plasmonic layer leads to even more complex scattering responses, beyond conventional Fano resonances, such as double and triple degenerate states, resembling an EIT-like response. Since the interference between the different states is very sensitive to the geometry, a plethora of different shades between Fano and EIT lineshapes can

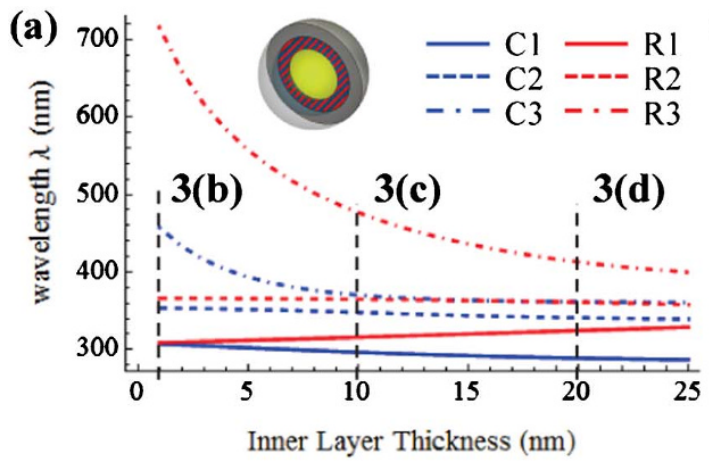

be obtained by simply modifying the aspect ratios of the multilayered nanoparticle. More layers may directly lead to even higher flexibility in terms of scattering manipulation, allowing exotic scattering signatures, such as the "Fano comb" spectrum discussed in Ref. 23. As expected, when material losses are considered, the Fanolike resonance linewidth slightly broadens and the difference between scattering peaks and dips becomes less pronounced. However, our results show that the dipolar nature of the involved phenomena leads to high robustness to realistic level of plasmonic material losses and, as a result, the peculiar shape of the scattering spectrum is preserved even after absorption is taken into account, which is an important feature towards the practical implementation and experimental verification of the proposed concepts.

These findings may have interesting applications in several fields of optics, ranging from signal processing to optical tagging, biological sensing and imaging. The proposed multilayered plasmonic nanoparticles may realize optical narrowband bandpass nanofilters ${ }^{33,34}$ whose response may be tailored with great flexibility. Moreover, since some of the scattering features outlined here, particularly those associated with surface plasmons supported at the outer interface, may be very sensitive to the background medium, these results may open interesting scenarios for biological and chemical sensing.

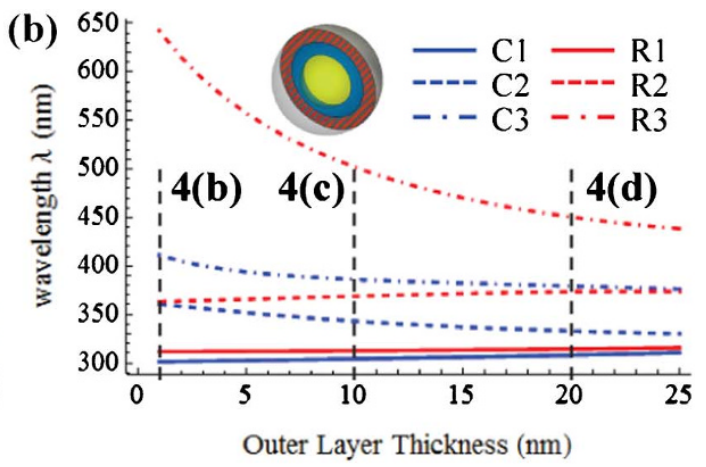

Figure 5 Frequency variation of cloaking and resonant scattering states versus the thickness of the plasmonic layers. Variation in wavelength of the cloaking dips (C1, C2, C3 blue lines) and resonant peaks (R1, R2, R3 red lines) as a function of the thickness of the nanoparticle's (a) inner layer and (b) outer layer. In both panels the geometry is shown in the inset and the layer under investigation is highlighted as a red striped surface. The vertical dashed lines correspond to the cases reported in panels (b), (c), (d) of Figs. 3 and 4, as indicated by the labels. 
Conversely, other scattering states, localized in the internal interfaces of the nanoparticle, may be very robust to the background medium and, for this reason, may be ideal to develop optical tagging platforms ${ }^{23}$ independent of the background, with several potential applications. For example, the extremely sharp narrowband scattering response, discussed in some of the previous examples, is very wellsuited for multicolor labeling in optical bio-imaging techniques ${ }^{35,36}$. The possibility to cover the visible and near-infrared frequency range with a large number of narrow resonance peaks may allow detecting multiple molecular targets at the same time, with great benefits, e.g., for tissue diagnosis. The flexibility of our approach for the manipulation of the scattering signature of subwavelength nanoparticles appears ideal to meet the requirements of all these applications.

\section{Methods}

All the results presented in this Report are obtained using analytical calculations based on full-wave Mie theory for scattering of spherical objects (for further details and physical insight, see, e.g., Ref. 17). As usually done, the impinging plane wave and the scattered fields are expanded in TE and TM spherical harmonics in a spherical coordinate system centered with the nanoparticle. By applying proper electromagnetic boundary conditions on each spherical interface, the scattering coefficients for $\mathrm{TE}$ and TM waves are obtained, which uniquely determine the fields everywhere. Total scattering cross section and field distributions are computed using Eq. (2) and related formulas, truncating the summations after reaching proper numerical convergence.

1. Maier, S. Plasmonics: Fundamentals and Applications. Springer, Berlin (2007).

2. Schuller, J. A., Barnard, E. S., Cai, W., Jun, Y. C., White, J. S. \& Brongersma, M. L. Plasmonics for extreme light concentration and manipulation. Nature Mater 9 , 193-204 (2010).

3. Luk'yanchuk, B., Zheludev, N. I., Maier, S. A., Halas, N. J., Nordlander, P., Giessen, H. \& Chong, C. The Fano resonance in plasmonic nanostructures and metamaterials. Nature Mater 9, 707-715 (2010).

4. Hao, F., Sonnefraud, Y., Dorpe, P. V., Maier, S. A., Halas, N. J. \& Nordlander, P. Symmetry Breaking in Plasmonic Nanocavities: Subradiant LSPR Sensing and a Tunable Fano Resonance. Nanolett. 8, 3983-3988 (2008).

5. Fan, J. A., Wu, C., Bao, K., Bao, J., Bardhan, R., Halas, N. J., Manoharan, V. N., Nordlander, P., Shvets, G. \& Capasso, F. Self-Assembled Plasmonic Nanoparticle Clusters. Science 328, 1135-1138 (2010).

6. Wu, C., Khanikaev, A. B., Adato, R., Arju, N., Yanik, A. A., Altug, H. \& Shvets, G. Fano-resonant asymmetric metamaterials for ultrasensitive spectroscopy and identification of molecular monolayers. Nature Mater 11, 69-75 (2012).

7. Miroshnichenko, A. E., Flach, S. \& Kivshar, Y. S. Fano resonances in nanoscale structures. Rev. Mod. Phys. 82, 2257-2298 (2010).

8. Zhang, S., Genov, D. A., Wang, Y., Liu, M. \& Zhang, X. Plasmon-Induced Transparency in Metamaterials. Phys. Rev. Lett. 101, 047401 (2008).

9. Liu, N., Langguth, L., Weiss, T., Kästel, J., Fleischhauer, M., Pfau, T. \& Giessen, H. Plasmonic analogue of electromagnetically induced transparency at the Drude damping limit. Nature Mater 8, 758-762 (2009).

10. Liu, N., Weiss, T., Mesch, M., Langguth, L., Eigenthaler, U., Hirscher, M., Sönnichsen, C. \& Giessen, H. Planar Metamaterial Analogue of Electromagnetically Induced Transparency for Plasmonic Sensing. Nanolett 10, 1103-1107 (2010).

11. Alù, A. \& Engheta, N. Achieving transparency with plasmonic and metamaterial coatings. Phys. Rev. E 72, 016623 (2005).

12. Alù, A. \& Engheta, N. Multifrequency Optical Invisibility Cloak with Layered Plasmonic Shells. Phys. Rev. Lett. 100, 113901 (2008).

13. Alù, A. \& Engheta, N. Cloaking a Sensor. Phys. Rev. Lett. 102, 233901 (2009).

14. Edwards, B., Alù, A., Silveirinha, M. G. \& Engheta, N. Experimental verification of plasmonic cloaking at microwave frequencies with metamaterials. Phys. Rev. Lett. 103, 153901 (2009).

15. Rainwater, D., Kerkhoff, A., Melin, K., Soric, J. C., Moreno, G., \& Alù, A. Experimental verification of three-dimensional plasmonic cloaking in free-space. New J. Phys. 14, 013054 (2012).

16. Fan, P., Chettiar, U. K., Cao, L., Afshinmanesh, F., Engheta, N. \& Brongersma, M. L. An invisible metal-semiconductor photodetector. Nature Photon 6 , 380-385 (2012).
17. Bohren, C. F. \& Huffman, D. R. Absorption and Scattering of Light by Small Particles. Wiley, New York (1983).

18. Alù, A. \& Engheta, N. Polarizabilities and effective parameters for collections of spherical nanoparticles formed by pairs of concentric double-negative, singlenegative, and/or double-positive metamaterial layers. J. Appl. Phys. 97, 094310 (2005).

19. Ruan, Z. \& Fan, S. Superscattering of Light from Subwavelength Nanostructures. Phys. Rev. Lett. 105, 013901 (2010).

20. Argyropoulos, C., Chen, P. Y., Monticone, F., D’Aguanno, G. \& Alù, A. Nonlinear Plasmonic Cloaks to Realize Giant All-Optical Scattering Switching. Phys. Rev. Lett. 108, 263905 (2012).

21. Mukherjee, S., Sobhani, H., Lassiter, J. B., Bardhan, R., Nordlander, P. \& Halas, N. J. Fanoshells: nanoparticles with built-in Fano resonances. Nanolett 10, 2694 (2010).

22. Wu, D., Jiang, S. \& Liu, X. Tunable Fano Resonances in Three-Layered Bimetallic $\mathrm{Au}$ and Ag Nanoshell. Journ. of Phys. Chem. C 115, 23797 (2011).

23. Monticone, F., Argyropoulos, C. \& Alù, A. Multi-Layered Plasmonic Covers for Comb-Like Scattering Response and Optical Tagging. under review, online at http://arxiv.org/abs/1210.4802

24. Argyropoulos, C., Chen, P. Y., D’Aguanno, G., Engheta, N. \& Alù, A. Boosting Optical Nonlinearities in $\varepsilon$-Near-Zero Plasmonic Channels. Phys. Rev. B 85, 045129 (2012).

25. Johnson, P. B. \& Christy, R. W. Optical Constants of the Noble Metals. Phys. Rev. B 6, 4370 (1972).

26. Campbell, G. A. Physical theory of the electric wave filter. Bell Systems Technical Journal 1, 1 (1922)

27. Foster, R. M. A reactance theorem. Bell Systems Technical Journal 3, 259 (1924).

28. Bohren, C. F. How can a particle absorb more than the light incident on it? Am. J. Phys. 51, 323 (1983).

29. Alù, A. \& Engheta, N. Higher-Order Resonant Power Flow Inside and Around Superdirective Plasmonic Nanoparticles. Journal of the Optical Society of America B 24, A89 (2007).

30. West, P. R., Ishii, S., Naik, G. V., Emani, N. K., Shalaev, V. M. \& Boltasseva, A. Searching for better plasmonic materials. Laser \& Photonics Reviews 4, 795 (2010)

31. Naik, G. V., Kim, J. \& Boltasseva, A. Oxides and nitrides as alternative plasmonic materials in the optical range. Opt. Mat. Express 1, 1090 (2011).

32. Pozar, D. M. Microwave Engineering. Wiley, New York (2004).

33. Engheta, N., Salandrino, A. \& Alù, A. Circuit Elements at Optical Frequencies: Nanoinductors, Nanocapacitors, and Nanoresistors. Phys. Rev. Lett. 95, 095504 (2005).

34. Engheta, N. Circuits with Light at Nanoscales: Optical Nanocircuits Inspired by Metamaterials. Science 317, 1698 (2007).

35. Chen, K., Liu, Y., Ameer, G. \& Backman, V. Optimal design of structured nanospheres for ultrasharp light-scattering resonances as molecular imaging multilabels. Journ. of Biomed. Optics 10, 024005 (2005).

36. Hu, R., Yong, K. T., Ding, H., Prasad, P. N. \& He, S. L. Tunable multicolored hybrid metallic nanoparticles for live human cancer cell imaging. J. Nanophoton 4 , 041545 (2010).

\section{Acknowledgments}

This work has been partially supported by AFOSR with the YIP award No. FA9550-11-1-0009 and the ONR MURI grant No. N00014-10-1-0942.

\section{Author contributions}

F.M. and C.A. conducted the calculations and collected the results. A.A. supervised the project and inspired the idea. All authors have contributed to writing the manuscript.

\section{Additional information}

Competing financial interests: The authors declare no competing financial interests.

License: This work is licensed under a Creative Commons

Attribution-NonCommercial-NoDerivs 3.0 Unported License. To view a copy of this license, visit http://creativecommons.org/licenses/by-nc-nd/3.0/

How to cite this article: Monticone, F., Argyropoulos, C. \& Alù, A. Layered plasmonic cloaks to tailor the optical scattering at the nanoscale. Sci. Rep. 2, 912; DOI:10.1038/ srep00912 (2012) 